Konrad-Zuse-Zentrum

für Informationstechnik Berlin

ARIE M.C.A. KOSTER

ADRIAN ZYMOLKA

MONIKA JÄGER

RALF HÜLSERMANN

CHRISTOPH GERLACH

\title{
Demand-wise Shared Protection for Meshed Optical Networks
}




\title{
Demand-wise Shared Protection for Meshed Optical Networks
}

\author{
Arie M. C. A. Koster* Adrian Zymolka* Monika Jäger $^{\dagger} \quad$ Ralf Hülsermann $^{\dagger}$ \\ Christoph Gerlach ${ }^{\dagger}$
}

\begin{abstract}
In this paper, a new shared protection mechanism for meshed optical networks is presented. Significant network design cost reductions can be achieved in comparison to the well-known 1+1 protection scheme. Demand-wise Shared Protection (DSP) bases on the diversification of demand routings and exploits the network connectivity to restrict the number of backup lightpaths needed to provide the desired level of protection. Computational experiments approve the benefits of the concept DSP for cost efficient optical network designs.
\end{abstract}

\section{Introduction}

The huge capacity of optical fibers is exploited by Wavelength Division Multiplexing (WDM). Individual wavelength channels build static bandwidth pipes to adjacent client network elements. As such, optical networks are replacing more and more SONET/SDH as transport layer technology. First generation optical networks deploy manually configured WDM systems. Meanwhile, the optical technology is leaving the pure transmission section of the network to progressively enter the switching function. Optical switching devices such as Optical Add Drop Multiplexers (OADM) and Optical Cross Connects (OXC) will be a key building block in future backbone networks. All-optical OXCs are fast and dispense the need of optical-to-electrical $(\mathrm{O} / \mathrm{E} / \mathrm{O})$ conversion. The $\mathrm{O} / \mathrm{E} / \mathrm{O}$ conversion is both costly and time-consuming. Carriers expect cost savings by reducing or eliminating the $\mathrm{O} / \mathrm{E} / \mathrm{O}$ conversion which is necessary in today's switching nodes. With the use of OADMs and OXCs, equipped with networking functions, the optical layer will evolve from a pure transmission layer to a real optical networking layer.

WDM optical networks concentrate an exponentially increasing amount of traffic in a single fiber. Therefore, survivability is a key concern in optical networks, as an innumerable

\footnotetext{
*Konrad-Zuse-Zentrum für Informationstechnik Berlin (ZIB), Takustraße 7, D-14195 Berlin, Germany. Email: \{koster,zymolka\}@zib.de

${ }^{\dagger}$ T-Systems Nova GmbH Technologiezentrum, Goslarer Ufer 35, D-10589 Berlin, Germany. Email: \{Monika.Jaeger,Ralf.Huelsermann,C.Gerlach\}@t-systems.com

Keywords: optical network design, shared protection, meshed optical networks, diversification

Mathematics Subject Classification (2000): 90B18, 68M10
} 
number of services are affected by a single fiber cut, the predominant form of failure in transport networks. Although higher network layers may provide their own recovery mechanisms, protection in the optical layer is advantageous especially in case of fiber cuts. The optical layer protection mechanism is close to the failure, i.e. the failure may be easily located and a large number of higher layer services are recovered by few protection switching operations in the optical layer.

Legacy transport networks have been deployed as rings because protection switching in ring topologies is simple and very fast. However, ring networks are limited in size (number of nodes). The advantages offered by ring networks are diminished when the size of the ring increases significantly so that difficult and costly inter-ring connections have to be installed. Mesh networks alleviate common problems associated with rings such as scalability and bandwidth inefficiency. They offer greater spatial diversity, shorter routing and eliminate the rigidity of the ring topologies.

The SONET/SDH network architecture is optimized for the transport and protection of voice traffic. It's Automatic Protection Switching (APS) capability provides the fault tolerance and reliability which is needed to support voice services. APS is a general term for three different protection switching schemes: 1+1, 1:1 and M:N APS, using different strategies for assigning protection resources. The most commonly used form of APS in SONET/SDH networks is $1+1$ dedicated path protection. Dedicated path protection is capacity inefficient since resource sharing is not possible. With the currently existing ring networks using dedicated path protection, more than half of the capacity is permanently assigned as spare capacity.

Protection in the optical layer is still in its infancy. In all-optical networks, sharing of resources is additionally hampered by the wavelength assigned to each transmission channel. First attempts to include reliability in the optical layer are therefore based on dedicated path protection mechanisms which again yield networks with low capacity efficiency.

In this paper, we introduce a new concept for the resource efficient design of survivable meshed optical networks. The concept applies protection sharing within single demands, and is therefore called Demand-wise Shared Protection (DSP). It is particularly suited for meshed optical networks, exploiting the connectivity in those networks as well as taking care of the specific routing requirements imposed on all-optical networks.

This paper is structured as follows. Section 2 describes the problem at hand and surveys some well-known survivability concepts. The newly developed concept DSP is introduced and discussed in Section 3. Its effectiveness for practical optical network design is illustrated in Section 4 by computational experiments. The paper is closed with some concluding remarks in Section 5.

\section{Survivability in optical networks}

In this section, we consider survivability concepts for optical networks. For this purpose, we initially give a detailed description of the design problem for optical networks. Then we review some well-known protection mechanisms related to the new concept DSP introduced in the subsequent section. 


\subsection{Problem specification}

We consider the problem of designing an optical network at minimum cost in order to satisfy given connection demands with specific survivability requirements. This task on the one hand includes the installation of hardware to provide sufficient transmission capacity on the links and switching capacity in the nodes. On the other hand, a routing of the asked connections has to be carried out, involving the use of spare capacity for protection.

Transmission capacity at a link is provided by operating WDM systems on optical fibers. Each fiber can carry a WDM system which offers a number of optical channels using different wavelengths. In the network nodes, optical cross-connects have to be installed to switch the channels optically. This enables to transmit traffic between a pair of nodes over one or several links by an ongoing optical connection which is called a lightpath. Setting up a lightpath needs to allocate an available wavelength channel on each crossed fiber and a switching port in each visited node. For exchanging the operated wavelength between two subsequent links, a wavelength converter has to be placed in the intermediate node.

To determine feasible hardware configurations for optical networks, several restrictions and further aspects have to be taken into account. Since we focus on survivability properties of the lightpath routing, these issues go beyond the scope of this paper. For a detailed description, we refer to [14].

The capacities provided by a suitable hardware configuration are used to realize the communication traffic which is given in terms of lightpaths. The number of lightpaths asked to connect a given pair of nodes is called a demand. We denote the total sum of all lightpaths to install as total demand. Each lightpath serves as transmission pipe for a high amount of traffic of several services. Since some services are offered with a performance guarantee on the optical layer, their operation has to be secured against disruption. Therefore, the associated lightpath must be protected in case a network failure occurs. As a consequence, a given number of lightpaths of each demand is asked to survive any single link or node breakdown (except of its end nodes). The remaining traffic is to be routed without protection precautions on the optical layer and is assumed to be protected in higher layers or

best effort traffic. To assert the stated survivability restriction, spare capacity is used for protection of the lightpaths by installing additional backup connections on disjoint paths. A precondition for implementing survivability is the sufficient connectivity of the network. A pair of nodes is called $k$ - (node) connected if it can be connected by $k$ paths which pairwise only share their end nodes. We assume that the network is at least 2-connected for each node pair asking for protected lightpath connections.

For the cost efficient design of optical networks, we aim at determining the cheapest hardware configuration which suffices for the routing of all lightpaths and their protection. Clearly, increasing capacity requirements cause increasing costs. Hence, network operators are in particular interested in resource efficient survivability concepts.

\subsection{Known survivability concepts}

The protection requirements are traditionally adapted for voice services, which need extremely short protection switching times and very high service availability. Existing trans- 
port networks mostly base on SDH rings. SDH protection is called Automatic Protection Switching (APS) and defines dedicated and shared protection architectures with 1+1, 1:1, 1:N, and M:N protection path mappings. All state-of-the-art SDH switching devices support $1+1$ path protection. Hence, incumbent service providers have integrated end-to-end $1+1$ path protection in their network operation, at the cost of using far more than $100 \%$ spare capacity for protection services. Since service providers need to reduce their costs, they are looking for ways to use their networks more efficiently. Shared protection mechanisms significantly reduce the required spare capacity by providing the same level of availability as dedicated protection.

Today's transport network design and configuration is done predominantly on the SDH layer. The optical layer only provides point-to-point transmission capacity between two SDH nodes. In future optical networks, comprising optical fibers, WDM systems, and all-optical cross-connects, protection mechanisms on the optical level will be introduced to ensure optical connections that cross several links and OXCs. As a result, the optical layer needs its own network design. In future optical networks, a large share of transport services will be protected in the optical layer, which will substitute much of today's SDH layer protection. Parts of the transport services, nevertheless will make use of higher layer protection or restoration functions.

There are two fundamentally different protection strategies. One approach is to protect signals by setting up complete backup paths from start to end nodes of the services. This strategy is called (end-to-end) path protection. Alternatively, backup paths may be set up only for individual segments (link or span) of the working path. The advantage of the latter strategy is that the protection switching is performed near the failure, with very short protection switching times, and no or little failure signaling effort. This is the reason why link or span protection is also called local protection. However, as a result, many backup paths are required for protecting a complete working path. In principle, all protection approaches are applicable for complete paths or segments of the path. However, in our case study we concentrate on path protection schemes only.

Dedicated backup path protection sets up two node-disjoint paths in the network, with an equal allocation of capacity reserved on both paths. The paths are routed on the shortest path and on the second shortest path, respectively, which is disjoint (link or span disjoint, node disjoint) from the shortest path. Alternatively, Suurballe's algorithm [3] can be applied which finds the shortest cycle in a network that contains the end nodes of the path. The shortest cycle is the working/backup path pair in the network with the least capacity, and avoids the problem that no disjoint second path can be found. There are two dedicated protection schemes:

- $1+1$ protection transmits for every single demand a signal duplicated on both paths. A switch at the end node selects the best signal. A dedicated path protection service promises $50 \mathrm{~ms}$ recovery time. It is very fast since only the tail end has to switch to the other signal in case of a failure. However, this protection scheme is very capacity intensive since resources are reserved and allocated prior to any failure.

- In 1:1 protection, two disjoint paths are set up with the same strategy as in $1+1$ protection, but the signal is only transmitted on the shortest path (working path). 
The second shortest path (protection path) is offered to best effort services. In case of failures, the interrupted service is switched from the working path to the preempted backup path. The strategy to allocate protection capacity on demand after a failure occurrence enables more efficient use of the network capacity, but it has higher protection switching times since the switching is not a local decision at the end node. The failure occurrence has to be detected and signaled in real-time to the service end nodes, which have to switch the signals to the backup path.

Usually, far more than $100 \%$ spare capacity is needed for dedicated path protection which is applied mostly in today's SDH ring networks as SDH 1+1 end-to-end APS. The routing of the two paths is very straightforward in ring networks. However, it is most capacity intensive, and as networks grow, protected inter-ring connections are complex and expensive. In mesh networks, dedicated path protection is more capacity efficient, since the routing is more diverse. A case study on dedicated path protection in WDM mesh networks is reported in [5].

1:1 protection is a special case of general M:N path mapping, where $\mathrm{M}$ protection paths are established to protect $\mathrm{N}$ different working paths between an ingress-egress node pair. M:N shared protection shares capacity in the sense that the spare resources are not dedicated for the recovery of a specific connection, but can be shared by the $\mathrm{N}$ connections for different failure scenarios. A more general shared path protection concept exists with a large number of strategies for sharing of backup resources between different services. Concepts for shared protection in optical networks have been proposed and evaluated, e.g. in [10, 6, 8, 9]. The basic principle is, that backup resources between any paths can be shared if services will not compete for the same resources at the same failure incidence. Shared protection can take advantage of mesh topology to reduce spare capacity consumption. In shared backup path protection the demands are routed along the shortest paths, and a single, fully disjoint backup path is pre-selected for each working path. Capacity is not pre-allocated to the backup paths, but the spare capacity on the backup routes is shared across working path demands that have no span in common, because backup capacity may not be needed simultaneously from different working paths (for single link or node failures). A real-time signaling phase seizes and cross-connects the shared capacity.

Rerouting differs fundamentally from the previous techniques, since it is based on a real-time calculation and establishment of the recovery path. A pool of spare capacities is provided which are allocated by individual recovery paths in failure cases. Case studies have shown that rerouting is very capacity efficient. One of the disadvantages of path restoration is that the recovery time can be quite long. Paths that need to be rerouted are recovered after a routing table update initiated following a failure.

\section{Demand-wise shared protection (DSP)}

For meshed optical networks, we introduce in this section a new survivability concept called Demand-wise Shared Protection (DSP). Its conceptual explanation is followed by a discussion of the properties, also in comparison to the known concepts surveyed in Section 2. Finally, we describe how the concept can be easily applied within any routing scheme as 
well as within our optimization tool for integrated planning of optical networks.

\subsection{The concept DSP}

As the name 'demand-wise shared protection' indicates, spare capacity is shared by the lightpaths belonging to a demand, but not among different demands. Hence, each demand has a spare capacity dedicated to it. By this, DSP tries to combine the advantages of both dedicated and shared protection. The concept bases on a routing scheme called diversification which was introduced in [4] and applied in [14].

Diversification aims at spreading the normal operation routing of a demand over several different paths. In context of optical networks, this spreading has to be done in integer amounts as lightpaths can not be splitted. For a demand of $d$ lightpaths, a parameter $0<\delta \leq 1$ is chosen to state an upper bound $\lfloor\delta d\rfloor$ on the fraction of the demand allowed to flow through a single link or node (of course, except of its source and destination). By this, any single component failure affects at most $\lfloor\delta d\rfloor$ lightpaths of the demand, while $d-\lfloor\delta d\rfloor$ lightpaths are ensured to survive. Note that the network has to be sufficiently connected since at least $\lceil d /\lfloor\delta d\rfloor\rceil$ node-disjoint paths are required. Thus, the connectivity $k$ of the source-destination pair poses on $\delta$ a natural lower bound of $\delta \geq \frac{1}{k}$.

Clearly, straightforward application of diversification can only limit the maximum loss incurred by any link or node breakdown. To provide protection against failures for a demand, additional backup lightpaths have to be installed. The idea of the proposed concept DSP consists in applying diversification on all working and backup lightpaths together. A clever spreading of this joint routing may significantly reduce the number of required backup lightpaths and hence save spare capacity.

Consider a demand of $d$ lightpaths of which $d^{*} \leq d$ have to be protected, $d, d^{*} \in \mathbb{Z}_{0}^{+}$. Let the source and destination be $k$-connected, $k \geq 2$. Instead of $d$ lightpaths, we route

$$
\tilde{d}=\max \left\{d,\left\lceil\frac{1}{1-\frac{1}{k}} d^{*}\right\rceil\right\}
$$

lightpaths with diversification parameter

$$
\delta=\frac{\tilde{d}-d^{*}}{\tilde{d}},
$$

where the value $\tilde{d}$ subsumes both working and protection lightpaths to be set up for this demand. Note that, $\delta \tilde{d}$ is integer. As a result, a routing of $\tilde{d}$ lightpaths with diversification parameter $\delta$ satisfies the survivability requirements, since in any failure situation, at least $\tilde{d}-\lfloor\delta \tilde{d}\rfloor=\tilde{d}-\delta \tilde{d}=d^{*}$ lightpaths survive. The given network connectivity allows for such a routing: by $\tilde{d} \geq \frac{1}{1-\frac{1}{k}} d^{*}$, we get $\delta \tilde{d}=\tilde{d}-d^{*} \geq \tilde{d}-\left(1-\frac{1}{k}\right) \tilde{d}=\frac{1}{k} \tilde{d}$ and hence $\delta \geq \frac{1}{k}$. So, the number of node-disjoint paths required is therefore at most

$$
\left\lceil\frac{\tilde{d}}{\lfloor\delta \tilde{d}\rfloor}\right\rceil=\left\lceil\frac{\tilde{d}}{\delta \tilde{d}}\right\rceil=\left\lceil\frac{1}{\delta}\right\rceil \leq\left\lceil\frac{1}{\frac{1}{k}}\right\rceil=k .
$$




\begin{tabular}{|c|c|c|c|c|c|c|c|c|c|c|c|c|c|c|c|c|}
\hline & $d$ & 1 & 2 & 3 & 4 & 5 & 6 & 7 & 8 & 9 & 10 & 15 & 20 & 25 & 35 & 50 \\
\hline$\frac{1}{3}$-protected & & & & & & & & & & & & & & & & \\
\hline $1+1 \mathrm{p}$ & otection & 1 & 1 & 1 & 2 & 2 & 2 & 3 & 3 & 3 & 4 & 5 & 7 & 9 & 12 & 17 \\
\hline & $k=2$ & 1 & 0 & 0 & 0 & 0 & 0 & 0 & 0 & 0 & 0 & 0 & 0 & 0 & 0 & 0 \\
\hline DSP & $k=3$ & 1 & 0 & 0 & 0 & 0 & 0 & 0 & 0 & 0 & 0 & 0 & 0 & 0 & 0 & 0 \\
\hline & $k=4$ & 1 & 0 & 0 & 0 & 0 & 0 & 0 & 0 & 0 & 0 & 0 & 0 & 0 & 0 & 0 \\
\hline$\frac{2}{3}$-protected & & & & & & & & & & & & & & & & \\
\hline $1+1 \mathrm{p}$ & otection & 1 & 2 & 2 & 3 & 4 & 4 & 5 & 6 & 6 & 7 & 10 & 14 & 17 & 24 & 34 \\
\hline & $k=2$ & 1 & 2 & 1 & 2 & 3 & 2 & 3 & 4 & 3 & 4 & 5 & 8 & 9 & 13 & 18 \\
\hline DSP & $k=3$ & 1 & 1 & 0 & 1 & 1 & 0 & 1 & 1 & 0 & 1 & 0 & 1 & 1 & 1 & 1 \\
\hline & $k=4$ & 1 & 1 & 0 & 0 & 1 & 0 & 0 & 0 & 0 & 0 & 0 & 0 & 0 & 0 & 0 \\
\hline full-protecte & & & & & & & & & & & & & & & & \\
\hline $1+1 \mathrm{p}$ & otection & 1 & 2 & 3 & 4 & 5 & 6 & 7 & 8 & 9 & 10 & 15 & 20 & 25 & 35 & 50 \\
\hline & $k=2$ & 1 & 2 & 3 & 4 & 5 & 6 & 7 & 8 & 9 & 10 & 15 & 20 & 25 & 35 & 50 \\
\hline DSP & $k=3$ & 1 & 1 & 2 & 2 & 3 & 3 & 4 & 4 & 5 & 5 & 8 & 10 & 13 & 18 & 25 \\
\hline & $k=4$ & 1 & 1 & 1 & 2 & 2 & 2 & 3 & 3 & 3 & 4 & 5 & 7 & 9 & 12 & 17 \\
\hline
\end{tabular}

Table 1: Number of backup lightpaths generated by $1+1$ protection and by DSP with different connectivity levels $k$.

Notice that $\tilde{d} \leq d+d^{*}$ holds in any case, where $d+d^{*}$ is the total number of working and backup lightpaths to be set up by $1+1$ protection. DSP takes advantage of the individual network connectivity for a demand and diversifies the routing of working and backup lightpaths together. Thereby, it reduces the potential impact of any failure and thus decreases the total number of required backup lightpaths. The following example demonstrates this effect.

Example: Assume a demand asks for $d=5$ lightpaths of which $d^{*}=3$ have to be protected, and the network provides a connectivity of $k=3$ between the source and destination of the demand. Then $\tilde{d}=\max \left\{5,\left\lceil\frac{1}{1-\frac{1}{3}}\right\rceil\right\}=\max \{5,5\}=5$, and $\delta=\frac{5-3}{5}=0.4$. So, DSP requires to use $\left\lceil\frac{5}{2}\right\rceil=3$ node-disjoint paths, on each at most 2 lightpaths routed. Note that $1+1$ protection would generate 8 lightpaths. For the same example with $d^{*}=4$, the proposed concept DSP asks for totally $\tilde{d}=6$ lightpaths, while $1+1$ protection installs in total 9 lightpaths.

The routing suggested in the example above also illustrates the principle of DSP by the diversification of failure risks. Balancing the lightpaths among $k$ node-disjoint paths for the normal operation routing implies that at most $\lceil d / k\rceil$ of them can be disturbed at once. Notice that for a fixed connectivity $k$, this value is minimal over all potential routings for the demand. Similarly, failures also influence the availability of the installed spare capacity. Thus, it should be diversified as well. The concept DSP spreads both working and backup lightpaths in combination. Thereby, the required number of backup lightpaths can be kept as low as possible. Table 1 provides for a range of demand values $d$ the number of backup lightpaths needed for $1+1$ protection (i.e., $d^{*}$ ) and for DSP (i.e., $\tilde{d}-d$ ) with different connectivities $k$. To simplify the illustration, the fraction of $d$ to be protected is chosen as $\frac{1}{3}, \frac{2}{3}$, and 1 , where the results are always rounded up (e.g., $d^{*}=\left\lceil\frac{1}{3} d\right\rceil$ ). 
In case of full protection $\left(d^{*}=d\right)$ and connectivity $k=2$, the concept DSP yields the same total number $\tilde{d}=2 d$ as $1+1$ protection. However, in meshed optical networks at least some source-destination pairs have connectivity $k>2$. For those demands, the total number $\tilde{d}$ of lightpaths to install is substantially lower than $2 d$. In fact, the possibility to set the parameters individually for each node-destination pairs allows to take advantage of the DSP concept even on sparse meshed topologies. Moreover, survivability requirements up to $d^{*}=\left\lceil\frac{k-1}{k} d\right\rceil$ are realized with no backup lightpaths at all, whereas for $1+1$ protection additional backup paths always have to be installed.

\subsection{Discussion of the concept DSP}

Related to general survivability principles, the concept DSP constitutes a mixing of dedicated and shared protection. While backup lightpaths are set up individually for each demand and thus the spare capacity is dedicated to it, the provided protection is shared among the working lightpaths within the demand. Hence DSP lies in between $1+1$ and shared protection.

Compared to $1+1$ protection, Table 1 shows that the number of required backup lightpaths can on the one hand be significantly reduced for meshed networks with higher connectivity. On the other hand, spreading the routing on node-disjoint paths for a demand yields in general longer lightpaths and thus increases the capacity needed for the normal operation routing. As a consequence, there is a trade-off between the total lightpath number to install and the total capacity allocated for them. Note that it is not necessary to fully exploit the connectivity for a demand. Instead, employing a lower connectivity leads to different DSP parameters, but also opens more flexibility for the lightpath routings. For instance, assuming $k=2$ for computing $\tilde{d}$ and $\delta$ typically yields more required backup lightpaths, but the routing is not forced to use long paths. These aspects are further highlighted in Section 4, where computational experiments reveal that the saving of lightpaths usually overcomes their increasing resource requirements.

A comparison to shared protection mechanisms is more difficult since they do not apply a common routing strategy. Although the general concept can be assumed to be resource efficient, this depends highly on the possibilities to identify and exploit disjointness relations. In fact, the shared use of spare capacity by the backup lightpaths for different working lightpaths must guarantee for the independence of the latter in failure situations. Hence, many relations between working lightpaths and available spare capacity have to be taken into account, which in particular complicates integrated planning and optimization. The concept DSP is simpler to apply for two reasons. First of all, only lightpaths within a demand are involved for disjointness considerations. Second, lightpaths which share backup capacity, always share the capacity from source to destination, not only one some links. Hence, switching at the nodes along the backup lightpaths can be preconfigured since it does not depend on the lightpath to be recovered. Moreover, such a complete routing for a demand can be computed efficiently, as described in the next subsection.

From the known concepts presented in Section 2, the most similar to DSP is M:N shared protection, which also protects a number of lightpaths commonly by some backup paths. However, M:N shared protection only regards the disjointness of the protected lightpath 
group and the associated backup paths, but does not take advantage of the network structure for the best possible spreading of the complete routing. The demand-wise exploitation of the provided connectivity allows DSP to choose a minimum number of backup lightpaths to realize the same level of protection.

From an operational point of view, the service recovery by DSP in case of a failure depends on the affected lightpath. Since additional backup lightpaths are set up for a demand, several signals can be transmitted doubled as for $1+1$ protection. In this case, a failure would simply require to switch to the surviving signal at the end node, resulting in very fast restoration times. Otherwise, if a working lightpath is not operated twice and is to be protected, a failure would require to reorganize (a part of) the demand's routing by allocating protected traffic to all survived lightpaths. This can be done by switching the protected signals at the source node to the surviving connections since all lightpaths are already installed. Nor a routing computation has to be performed neither lightpaths have to be set up on request. As a result, the restoration mechanism reduces on switching operations in the end nodes, providing fast fault recovery.

\subsection{Optical network design with DSP}

To apply DSP, the routing of a demand has to be computed with respect to the concept parameters. For a single demand, this can be modeled as a min-cost flow problem. For each link and each node (except of the demand's end nodes), an upper bound on the flow is given by the diversification bound. Each feasible flow of the demand's value according to these capacity bounds corresponds to a possible complete lightpath routing for this demand. Among those feasible lightpath routings, a min cost flow can be determined by specifying an appropriate cost function. A cost of 1 per capacity unit on each link yields a demand routing with minimum total number of hops, whereas assigning link lengths as costs results in a routing with minimum total length. Combinations of the above and other linear cost functions can be specified without loss of generality. Since min-cost flow problems are polynomial solvable (cf. [1]), a complete demand routing based on DSP can be achieved efficiently this way. Moreover, it can be easily integrated within any sequential routing algorithm.

The consideration of lightpath routings for multiple demands simultaneously leads in general to a better utilization of the network resources. Such lightpath routings can be computed by use of (mixed-integer) linear programming. To apply DSP, additional constraints have to be inserted to satisfy the diversification flow bounds. Without going into detail, these constraints have a simple structure since they only involve the flow of a single demand. In addition, their number is polynomially bounded, so the size of the program does not increase much. In contrast, sharing resources between different demands is much harder to model in such approaches since path disjointness for any failure situation has to be taken into account.

Finally, DSP can also be incorporated similarly into integrated planning methods that aim at performing the dimensioning of capacities and the demand routing in a single step. One such tool is Optical Network Design (OND) described in [14] which applies state-of-the-art mathematical optimization methods based on integer programming. Solely adapting the 
demand value and diversification parameter suffices to incorporate DSP in the optimization model. This adaption has in fact been used for the computational experiments to evaluate the potential of DSP. Due to lack of space, we refer to [14] for this formulation.

\section{Computational experiments}

To reveal the benefits of DSP, we present two computational studies in this section. The first study discusses the direct advantages of the new protection mechanism in comparison with the $1+1$ protection in state-of-the-art network design tools. The second study shows further potential of DSP for the design of optical networks.

\subsection{Setting and instances}

For the comparisons, we relied on two software solutions for optical network design. The results for $1+1$ protection have been generated with the use of WDM Guru, a commercial network planning tool of OPNET [12]. For a given topology, cost model, and traffic demand matrix, the tool designs a $1+1$ protected, cost effective transparent optical network configuration. The new concept has been implemented within the Optical Network Design (OND) tool presented in [14], where a component-based mathematical optimization model for integrated optical network design is proposed. The model represents the dimensioning, routing, and wavelength assignment for transparent optical networks with wavelength conversion. It includes optical fibers, WDM systems, OXCs, and wavelength converters (of single optical signals $)^{1}$. OND applies integer programming techniques [11] to compute a cost efficient network configuration. In addition, OND outputs a lower bound on the total cost of any design that satisfies the prerequisites. OND relies on DISCNET [13] and uses CPLEX [7] as linear programming solver.

Both the network data and the cost model have been provided by T-Systems Nova. The cost model bases on realistic figures, normalized by an appropriate scaling factor. For the purpose of this paper, we use a simpler optical component model than in [14]. We consider one type of fiber, one type of WDM system offering 40 wavelength channels, one type of wavelength converter, and two types of OXCs with 64 and 128 bidirectional ports, respectively. A small OXC costs 193.2, a big OXC 312.4, a WDM system 24, and a wavelength converter 2. The fiber cost composes of two parts: a cost of 0.05 per $\mathrm{km}$ for the fiber itself, and a cost of 6 for regeneration (by EDFA's) after each link segment of $70 \mathrm{~km}$.

The computational results have been carried out on a hypothetical 17-node instance which reflects a typical German optical backbone network, as schematized in Figure 1. The network has 26 links, and a total of 686 lightpaths have to be routed during normal operation. The in total 57 source-destination pairs are composed of 38 pairs that are 2-connected, 18 pairs that are 3 -connected, and 1 pair that is even 4 -connected. The traffic demands have been derived from a German population distribution model as described in [2] and are based on a conservative estimation for the year 2000. Although the level of protection

\footnotetext{
${ }^{1}$ Note that, WDM Guru does not allow the installation of wavelength converters. Hence, its routing is carried out without using conversion.
} 


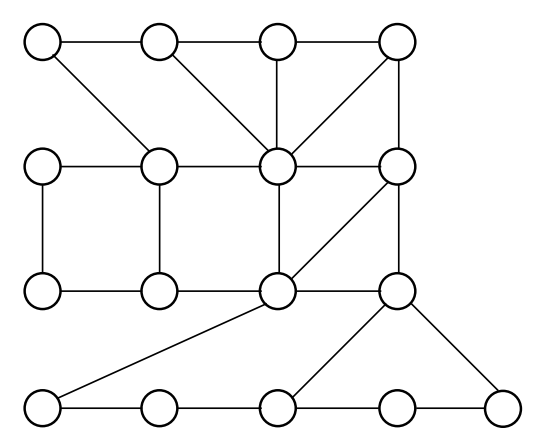

Figure 1: The 17-node network used for computational experiments.

can be stated individually for every demand, in this study we consider four scenarios where the level of protection is uniform for all demands. The four scenarios are defined by the percentage of the lightpaths that should survive a failure of a node or link and are denoted UnPRotected, $\frac{1}{3}$-PRotected, $\frac{2}{3}$-PRotected, and FUll PRotected. The scenario UNPROTECTED is provided for reference only. For a demand of $d$ lightpaths, in the scenarios $\frac{1}{3}$-PROTECTED and $\frac{2}{3}$-PROTECTED respectively $\left\lceil\frac{1}{3} d\right\rceil$ and $\left\lceil\frac{2}{3} d\right\rceil$ lightpaths have to survive a node or link failure (except for failure of its source or destination). The total number of lightpaths to survive in each scenario as well as the total number of lightpaths precomputed for the different protection mechanisms are listed in Table 2. For the concept DSP, we differentiate between the case that 2-connectivity is exploited for all demands and the case that the maximum connectivity available for each demand is exploited. The mentioned reductions are calculated for the total number of lightpaths; restricted to the backup lightpaths, this value is significantly higher (up to $95 \%$ compared to $1+1$ protection). Note that, for scenario FULL PROTECTED in case of 2-connectivity the total number of lightpaths to route equals the number of $1+1$ protection (as pointed out in Section 3). Also note that for scenario $\frac{1}{3}$-PROTECTED the total number of lightpaths does not differ between 2-connectivity and maximum connectivity since $\tilde{d}$ attains the same maximum for all $d$, see Table 1 . By Table 1 , we also can derive that 13 demands have value 1 , since only those demands cause an increase of $\tilde{d}$ in comparison with $d$ for $\frac{1}{3}$-PROTECTED.

\subsection{DSP vs. $1+1$ protection}

In our first study, we compare the network cost of $1+1$ protection computed by WDM Guru with those computed by OND for the max-connectivity and 2-connectivity exploitation of DSP. Table 3 provides the cost for the network, split into node, link, and total cost for each scenario and both protection mechanisms, where DSP exploits the maximum connectivity for each demand. The column "opt.gap" refers to the difference between the total cost and the lower bound provided by OND on the total unavoidable cost, i.e., it is guaranteed that no network configuration exists that undersells the cost with more than the percentage listed. The last column shows the potential savings of DSP in comparison to $1+1$ protection.

Table 3 shows that potential savings up to $20 \%$ can be achieved by application of the concept DSP, compared to $1+1$ protection. For the scenarios $\frac{1}{3}$-PROTECTED and $\frac{2}{3}$-PROTECTED, 


\begin{tabular}{l|rr|r|rr|rr}
\hline & total demand & demand to survive & 1+1 protection & \multicolumn{4}{|c}{ Demand-wise Shared Protection } \\
instance & (\# lightpaths) & (\# lightpaths) & total to route & 2-conn. & reduction & max-conn. & reduction \\
\hline UNPROTECTED & 686 & 0 & 686 & 686 & $0.0 \%$ & 686 & $0.0 \%$ \\
$\frac{1}{3}$-PROTECTED & 686 & 254 & 940 & 699 & $25.6 \%$ & 699 & $25.6 \%$ \\
$\frac{2}{3}$-PROTECTED & 686 & 478 & 1164 & 956 & $17.9 \%$ & 835 & $28.3 \%$ \\
FULL PROTECTED & 686 & 686 & 1372 & 1372 & $0.0 \%$ & 1191 & $8.7 \%$ \\
\hline
\end{tabular}

Table 2: Number of lightpaths to route by application of the various protection mechanisms.

\begin{tabular}{l|rrr|rrrr|r}
\hline & \multicolumn{4}{|c|}{ 1+1 protection } & \multicolumn{2}{c|}{ Demand-wise Shared Protection (max-conn.) } & comparison \\
instance & node cost & link cost & total cost & node cost & link cost & total cost & opt.gap(\%) & savings(\%) \\
\hline UNPROTECTED & 6186.40 & 1465.20 & 7651.60 & 6186.40 & 1436.25 & 7622.65 & 11.0 & 0.0 \\
$\frac{1}{3}$-PROTECTED & 8225.20 & 2405.00 & 10594.30 & 6663.20 & 1716.65 & 8379.85 & 13.2 & 20.9 \\
$\frac{2}{3}$-PROTECTED & 10917.60 & 2963.45 & 13881.05 & 8895.20 & 2623.20 & 11518.40 & 6.5 & 17.0 \\
FULL PROTECTED & 12093.20 & 3644.40 & 15737.60 & 11854.80 & 3477.25 & 15332.05 & 6.4 & 2.6 \\
\hline
\end{tabular}

Table 3: Results in terms of (total) network costs for max-connectivity. 
the disadvantages of probably longer paths by the diversification requirement are amply compensated by the component savings due to the smaller number of backup lightpaths to be reserved (cf. the reductions in number of lightpaths in Table 2). In the FULL PROTECTED case, the reduction of capacity requirements lowers the total cost by a smaller amount. Moreover, relating the results for $\frac{1}{3}$-PROTECTED and $\frac{2}{3}$-PROTECTED shows that a protection level increase from $\frac{1}{3}$ to $\frac{2}{3}$ for all demands can be realized with less than $9 \%$ additional cost (11518.4 vs. 10594.3) by changing from $1+1$ protection to DSP (and similarly for upgrading $\frac{2}{3}$ to full protection).

A disadvantage of exploiting max-connectivity for DSP can be the increasing length of the node-disjoint paths. Consider for example the three node-disjoint paths between the two nodes at the upper right corner of the 17-node network in Figure 1. The third path, besides the direct link between the nodes and the path across the common neighbor, goes across 7 links, thereby consuming many resources. In such a case, exploitation of 2-connectivity instead of 3-connectivity can be beneficial since such long paths are not necessary anymore (which does not imply that they can not be chosen). On the other hand, the number of required backup lightpaths increases if less connectivity is exploited (cf. Table 2). To determine the impact of maximum connectivity in comparison with 2-connectivity, we applied the same computations for $k=2$. Table 3 provides the results for this case. Note that the results for WDM Guru are the same as well as those for the scenarios UNPROTECTED and

$\frac{1}{3}$-PROTECTED, since the total number of routed lightpaths has not changed.

Compared to the maximum connectivity case, the total cost increases with about $1 \%$, whereas the total number of lightpaths is about $15 \%$ higher. So, to reduce the number of long lightpaths in the network, it can be beneficial to restrain the exploited connectivity to 2. On the other hand, an additional cost saving can be achieved by exploiting the maximum connectivity. It is likely that an individual ruling of the demands concerning the exploited connectivity is most beneficial with respect to the total network cost. Compared to WDM Guru, still significant savings can be achieved by the exploitation of the new concept for scenario $\frac{2}{3}$-PROTECTED. For FULL PROTECTED the savings are marginal since the same number of lightpaths has to be routed.

\subsection{The benefit of additional connectivity}

Our second study aims to show further prospects of the presented survivability concept. In the first study, we applied the maximum connectivity for every demand to limit the number of backup lightpaths to be routed. In particular for high protection levels, the number of backup lightpaths can be further decreased by increasing the connectivity of the network. In the network of Figure 1, many node pairs are only 2-connected. Increasing this connectivity to 3 or even 4 reduces the number of needed backup lightpaths, cf. Table 1. To increase connectivity requires the installation of new links which clearly causes cost. The implementation of DSP within OND allows for an analysis of the potential savings induced by new links. A comparison of these savings against necessary investments enables to identify profitable network extensions.

To illustrate this analysis, we added two links to the network of Figure 1, see Figure 2. Their insertion increases the connectivity for a total of 9 demands and causes a reduction 


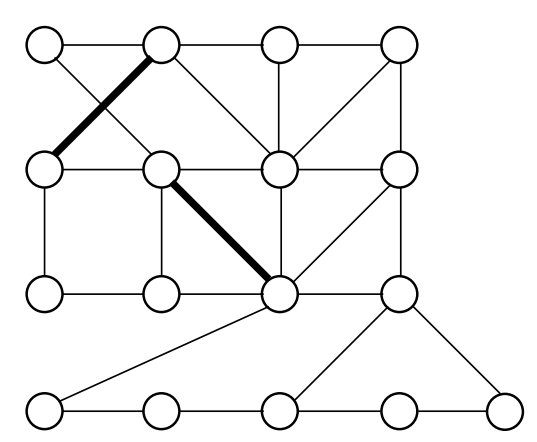

Figure 2: The 17-node network including two additional links.

in the total number of lightpaths to route of about $5 \%$. Table 5 shows the results obtained for the scenarios $\frac{2}{3}$-PROTECTED and FULL PROTECTED (the number of backup lightpaths is not affected by the increased connectivity in the other scenarios).

Compared to the corresponding results in Table 2 and 3 , a decrease in both the node and link cost can be observed. This indicates that in fact the average hop distance of the lightpaths declines. By this, additional cost savings could be achieved, in addition to the reduction of the number of lightpaths to be routed, cf. the results for scenario FULL PROTECTED.

Potentially, all network extensions can be analyzed this way to find the best trade-off between investments and savings. As alternative, fixing a budget for possible network extensions limits the number of scenarios that has to be investigated.

\section{Conclusions}

In this paper, we introduced the concept of Demand-wise Shared Protection (DSP) to deploy reliable optical networks. DSP exploits the connectivity available in meshed networks. Based on diversification, DSP spreads the joint routing of working and backup lightpaths. Thereby, the number of backup paths can be kept as low as possible, which saves spare capacity. Hence, the concept DSP is more resource efficient than dedicated protection mechanisms without losing fast fault recovery. In addition, compared with known shared protection mechanisms, the computation of resource efficient routings is simpler.

Further research seems to be worthwhile in several directions. On the one hand, possible extensions of the concept to resource sharing among different demands without losing the benefits of dedicated protection mechanisms should be considered. On the other hand, a more detailed analysis of the underlying connectivity and the length of the lightpath routings could be used for concept refinements. Finally, the adaption of the concept DSP for use in dynamic environments should be studied. 


\begin{tabular}{l|rrrr|rr}
\hline & \multicolumn{3}{|c|}{ Demand-wise Shared Protection (2-conn.) } & \multicolumn{2}{c}{ savings compared to } \\
instance & node cost & link cost & total cost & opt.gap(\%) & 1+1 protection(\%) & max-conn.(\%) \\
\hline UNPROTECTED & 6186.40 & 1436.25 & 7622.65 & 11.0 & 0.0 & 0.0 \\
$\frac{1}{3}$-PROTECTED & 6663.20 & 1716.65 & 8379.85 & 13.2 & 20.9 & 0.0 \\
$\frac{2}{3}$-PROTECTED & 9207.60 & 2437.75 & 11675.35 & 12.7 & 15.9 & -1.4 \\
FULL PROTECTED & 12092.40 & 3390.15 & 15482.55 & 6.4 & 1.6 & -1.0 \\
\hline
\end{tabular}

Table 4: Results in terms of (total) network costs for using only 2-connectivity.

\begin{tabular}{l|rrrrr|rr}
\hline \multirow{2}{*}{ instance } & \multicolumn{4}{|c|}{ Demand-wise Shared Protection - 2 new links } & \multicolumn{3}{|c}{ reduction of } \\
& total to route & node cost & link cost & total cost & opt.gap $(\%)$ & lightpaths(\%) & $\operatorname{costs}(\%)$ \\
\hline$\frac{2}{3}$-PROTECTED & 794 & 8567.60 & 2400.20 & 10967.80 & 9.3 & 4.9 & 4.8 \\
FULL PROTECTED & 1120 & 11557.60 & 3195.75 & 14753.35 & 8.5 & 6.0 & 9.6 \\
\hline
\end{tabular}

Table 5: Results in terms of (total) network costs after introducing two new links. 


\section{References}

[1] R. K. Ahuja, T. L. Magnanti, and J. B. Orlin. Network Flows: Theory, Algorithms, and Applications. Prentice Hall, New Jersey, 1993.

[2] A. Betker and C. Gerlach. Traffic demand model for dynamic optical transport networks based on the population distribution. In 4. ITG Fachtagung Photonische Netze, Leipzig, 2003.

[3] R. Bhandari. Survivable Networks, Algorithms for Diverse Routing. Kluwer Academic Publishers, Boston/Dordrecht/London, 1999.

[4] G. Dahl and M. Stoer. A cutting plane algorithm for multicommodity survivable network design problems. INFORMS Journal on Computing, 10(1):1-11, 1998.

[5] S. De Patre, G. Maier, M. Martinelli, and A. Pattavina. Design of static WDM mesh networks with dedicated path-protection. In Next Generation Optical Network Design and Modelling, pages 281-294. Kluwer Academic Publishers, Boston/Dordrecht/London, 2003.

[6] A. Groebbens, D. Colle, C. Develder, S. de Maessschalck, M. Pickavet, and P. Demeester. Use of backup trees to improve resource efficiency of MPLS protection mechanisms. In Proc. of Design of Reliable Communication Networks Workshop (DRCN) 2001, pages 152-159, Budapest, Hungary, 2001.

[7] ILOG. CPLEX version 7.5, 2001. http://www.ilog.com/products/cplex.

[8] B.G. Josza and D. Orincsay. Shared backup path optimization in telecommunication networks. In Proc. of Design of Reliable Communication Networks Workshop (DRCN) 2001, pages 251-257, Budapest, Hungary, 2001.

[9] H. Kerivin, B. Liau, and T.-T.-L. Pham. Survivable capacitated networks - Comparison of shared protection mechanisms. In Proc. Of Networks 2002, pages 379-388, Munich, Germany, 2002.

[10] C. Mauz. Allocation of spare capacity for shared protection of optical paths in transport networks. In Proc. of Design of Reliable Communication Networks Workshop (DRCN) 2001, pages 22-27, Budapest, Hungary, 2001.

[11] G. L. Nemhauser and L. A. Wolsey. Integer and Combinatorial Optimization. Wiley, N.Y., 1988.

[12] OPNET. WDM Guru. http://www.opnet.com.

[13] R. Wessäly. DImensioning Survivable Capacitated NETworks. PhD thesis, Technische Universität Berlin, 2000.

[14] A. Zymolka, A. Koster, and R. Wessäly. Transparent optical network design with sparse wavelength conversion. In Proc. ONDM 2003, Budapest, Hungary, February 2003. 